\title{
Diagnóstico e intervención temprana de insuficiencia velofaringea posterior a palatoplastia primaria en pacientes atendidos interdisciplinariamente en FISULAB [Bogotá, Colombia]
}

\section{Diagnosis and early intervention of velofaringeal insufficiency posterior to primary palatoplasty in patients attended interdisciplinarily in FISULAB [Bogotá, Colombia]}

José Rolando PRADA*, Juan Carlos ZAMBRANO*, Diana M. DÍAZ-LÓPEZ**

María del Pilar ECHEVERRI ${ }^{\star \star \star}$, Catalina TIBAQUIRA ${ }^{* \star *}$, Ana María PARRA ${ }^{\star \star * *}$

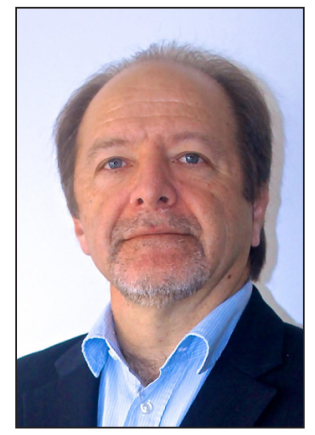

Prada, J.R.

\section{Resumen}

Introducción y Objetivo. El tratamiento integral e interdisciplinario de los pacientes con paladar hendido es fundamental para garantizar resultados a largo plazo. El habla es una de las metas principales en el tratamiento ya que permitirá un adecuado aprendizaje escolar y socialización. La insuficiencia velofaríngea (IVF) se debe a alteraciones anatómicas del esfínter velofaringeo y el manejo temprano y adecuado de los pacientes con paladar hendido es clave para evitarla.

El objetivo de este estudio es determinar el porcentaje de IVF detectado y manejado antes de los primeros 5 años de vida en pacientes con paladar hendido tratados interdisciplinariamente en el Centro de Rehabilitación para Niños con Labio y Paladar Fisurado (FISULAB) en Bogotá, Colombia, evidenciando la efectividad del abordaje temprano.

Material y Método. Estudio descriptivo tipo serie de casos en el que presentamos los resultados funcionales del tratamiento continuo en pacientes con paladar hendido, diagnosticados y tratados de forma exclusiva en FISULAB de acuerdo a su guía integral. Del total de 972 pacientes atendidos en la institución, 118 cumplían con los criterios de inclusión.

Resultados. Estudiamos 118 pacientes con paladar hendido con o sin labio hendido atendidos bajo la guía integral desde el nacimiento hasta los 5 años de edad: 67 varones y 51 mujeres. Del total, 17 (14.4\%) presentaron IVF ( 8 varones y 9 mujeres). De los que presentaron IVF, 4 (23.5\%) tenían diagnóstico sindrómico asociado. La incidencia de IVF en nuestra población de pacientes, excluyendo los pacientes sindromáticos, fue del $11 \%$, con una resolución del $100 \%$ de la IVF en los pacientes que fueron llevados a uno o múltiples procedimientos quirúrgicos.

Conclusiones. El diagnóstico temprano y el manejo interdisciplinario de la IVF son esenciales para asegurar el éxito en la rehabilitación del lenguaje de los pacientes con paladar hendido. Un tratamiento conservador y por etapas de la IVF puede asegurar buenos resultados minimizando complicaciones.

Palabras clave Insuficiencia velofaríngea, Paladar hendido, Palatoplastia.
Nivel de evidencia científica Recibido [esta versión] Acentado 4c Terapéutico

3 marzo/2019
21 enero/2019 are essential to ensure the success in speech rehabilitation in patients with cleft palate. A conservative and stepwise treatment of VPI has shown good results, minimizing complications

Background and Objective. A comprehensive and interdisciplinary treatment of patients with cleft palate is essential to ensure satisfactory longterm results. Adequate speech is one of the main goals because it allows each patient a proper adjustment to their educational and social environment. Velopharyngeal insufficiency (VPI) occurs due to anatomic changes of the velopharyngeal sphincter. Early and appropriate management of patients with cleft palate is crucial to prevent velopharyngeal insufficiency.

The aim of this study is to determine the incidence of patients with VP that were detected and received treatment during their first 5 years of age at FISULAB (Rehabilitation Center for Children with Cleft lip and Palate), Bogota, Colombia, and to evaluate the effectiveness early treatment.

Methods. Descriptive study of case series. Patients included were diagnosed with cleft palate and treated exclusively in FISULAB, following the treatment protocol of this institution. A total of 972 patients were treated and 118 patients met the inclusion criteria.

Results. A total of 118 patients with cleft palate, with or without associated cleft lip, were treated following the institutional protocol: $17(14.4 \%)$ had VPI ( 8 male and 9 female). Of the patients who had VPI, 4 (23.5\%) had a syndromic diagnosis. The incidence of VPI in our patient population was $11 \%$ with a resolution of $100 \%$ of VPI after surgical treatment.

\section{Key words Velopharyngeal insufficiency, Cleft palate, Palatoplasty.}

\section{Level of evidence Received [this version] Accepted} 4c Therapeutic

Conflicto de intereses: los autores declaran no tener ningún interés financiero relacionado con el contenido de este artículo. Financiación: No hubo fuentes externas de financiación para este trabajo.

\footnotetext{
Especialista en Cirugía Plástica y Craneofacial, Servicio de Cirugía Plástica del Hospital Infantil de San José y Centro de Rehabilitación para Niños con Labio y Paladar Fisurado (FISULAB), Bogotá, Colombia.

** Especialista en Cirugía Plástica, Hospital Infantil de San José, Bogotá, Colombia.

*** Fonoaudióloga del Centro de Rehabilitación para Niños con Labio y Paladar Fisurado (FISULAB), Bogotá, Colombia.

**** Medico General, Centro de Rehabilitación para Niños con Labio y Fisurado (FISULAB), Bogotá, Colombia.
} 


\section{Introducción}

El tratamiento integral de los pacientes con paladar hendido es fundamental para garantizar la calidad y continuidad de los cuidados a lo largo de su vida. De forma ideal, la evaluación inicial se debe realizar en las primeras semanas de vida por un grupo interdisciplinario, asegurando la creación de un vínculo entre la familia y el equipo de especialistas a fin de obtener así mejores resultados clínicos y estéticos para el paciente. Sin embargo, debemos tener presente que a pesar de un adecuado tratamiento para la corrección de esta patología, los pacientes con paladar hendido pueden presentar trastornos del habla después de la primera corrección quirúrgica, que de no ser corregidos, pueden afectar significativamente a la comunicación y al desarrollo del lenguaje. ${ }^{(1)}$

$\mathrm{La}$ incidencia de insuficiencia velofaríngea (IVF) posterior a palatoplastia primaria se encuentra en un rango del 5-36\% en distintas series publicadas, convirtiéndose en un reto clínico para los cirujanos plásticos. ${ }^{(2)} \mathrm{La}$ IVF es un trastorno estructural que genera alteraciones del habla y el término se emplea para describir una variación anormal de la función velofaríngea de etiología estructural como resultado de un cierre defectuoso del esfinter velofaríngeo durante el habla, lo que permite que el aire escape a través de la nariz en lugar de por la boca. ${ }^{(3)}$

La insuficiencia velofaríngea puede producirse por alteraciones anatómicas como hendiduras, paladar abierto, paladar submucoso o postquirúrgico por espacios residuales, interferencia mecánica como las adenoides, amígdalas o bridas en los pilares, por desproporción palatofaringea como ocurre en el paladar corto o en las lesiones ablativas. ${ }^{(4)}$

El esfinter velofaríngeo tiene un componente velar y uno faríngeo; el movimiento del componente velar se produce por la acción del musculo levator veli palatini y el movimiento del componente faríngeo depende de la contracción del constrictor de la faringe y del músculo palatofaríngeo. Los músculos del paladar (levator veli palatini, tensor veli palatini y uvularis) trabajan en conjunto con los músculos palatofaríngeo, constrictor de la faringe y palatogloso para producir el cierre velofaríngeo. ${ }^{(5)}$

Existen diversos patrones de cierre velofaríngeo que fueron descritos inicialmente por Skolnick y Croft, quienes consideraron los patrones de cierre coronal, sagital, circular y circular con rodete. ${ }^{(6,7)}$ Un estudio realizado por Prada y col. determinó que en la población de Bogotá, el patrón predominante es el circular con un 55\% de presentación, seguido del coronal con un $30 \%$ y del circular con rodete con un $15 \%$, proporción que se mantiene en los pacientes con secuelas de paladar hendido. ${ }^{\left({ }^{8}\right)}$
La fundación FISULAB (Centro de Rehabilitación para Niños con Labio y Paladar Fisurado), en Bogotá, Colombia, nuestro principal centro de estudio, es una institución de referencia para pacientes con labio y paladar hendido (LPH) en el país y se caracteriza por realizar el tratamiento integral de los pacientes con LPH, permitiendo su seguimiento desde el momento del diagnóstico y a través de todos los procesos diagnósticos y terapéuticos requeridos a lo largo de su vida.

En el presente estudio mostramos los resultados obtenidos en cuanto a incidencia de IVF en nuestra población de pacientes atendidos en FISULAB desde el nacimiento hasta los 5 años de vida, así como el manejo recibido en dichos casos y el porcentaje de corrección después de los procedimientos realizados.

\section{Material y método}

Realizamos un estudio descriptivo de tipo serie de casos con los pacientes de labio y paladar hendido tratados exclusivamente en FISULAB. Consideramos como criterios de inclusión: pacientes con paladar hendido con o sin labio hendido asociado, tratados desde el inicio de forma exclusiva en FISULAB, con seguimiento mínimo de 5 años desde el primer procedimiento. Todos recibieron manejo integral y seguimiento interdisciplinario desde el inicio del tratamiento por Cirugía Plástica, Fonoaudiología, Otorrinolaringología, Psicología, Odontología y Trabajo Social.

En estos pacientes evaluamos en el tiempo la efectividad de la palatoplastia primaria mediante la determinación del porcentaje de IVF posterior a la misma. El diagnóstico de IVF se realizó mediante la evaluación clínica, fonológica y videonasoendoscopia. Los pacientes a quienes se les realizó diagnóstico de IVF fueron llevados a corrección quirúrgica mediante diversas técnicas según la alteración encontrada en la nasoendoscopia; sin embargo, de persistir los signos de IVF o alteraciones en el habla, se realizó una segunda nasoendoscopia para confirmar la presencia de IVF residual, la cual una vez diagnósticada pudo requerir de un procedimiento quirúrgico adicional y de una nasoendoscopia postoperatoria para confirmar el porcentaje de reducción de IVF.

Consignamos la información obtenida en una base de datos Excell y la analizamos mediante el programa STATA 12.0, sometiendo los datos a análisis mediante estadística descriptiva.

\section{Resultados}

De 972 pacientes elegibles con labio y paladar hendido atendidos en FISULAB, 118 cumplieron los criterios 
de inclusión al tener diagnóstico de paladar hendido con o sin labio hendido y ser pacientes atendidos desde su nacimiento hasta mínimo los 5 años de edad exclusivamente en FISULAB. Del total de pacientes, 15 (12.7\%) tenían diagnóstico de paladar hendidos aislado y 103 (87.2\%) de labio y paladar hendido. De esta población, 67 pacientes $(56,7 \%)$ eran de sexo masculino y $51(43.2 \%)$ femenino. Adicionalmente 19 (16.1\%) eran pacientes sindrómicos y 99 (83.8\%) no sindrómicos (Tabla I). Todos los pacientes fueron llevados a palatoplastia primaria por los 2 cirujanos plásticos de la institución.

El promedio de edad en que se realizó la palatoplastia primaria fue en su mayoría entre los 8 y los 10 meses de edad (52.9\%, 9 casos), en un segundo grupo entre los 11 a 15 meses $(29.4 \%, 5$ casos) y en 3 casos en pacientes mayores a 16 meses de edad (17.6\%). La técnica de palatoplastia primaria más empleada fue la de Bardach y en segundo lugar la de Furlow.

Tras la palatoplastia primaria, 101 pacientes $(85.5 \%)$ presentaron habla normal durante el período de seguimiento, y 17 (14.4\%) presentaron IVF (8 de sexo masculino y 9 de sexo femenino), 4 (23.5\%) de los cuales tenían diagnóstico sindrómico de base, 2 con diagnóstico de Secuencia de Pierre Robin, 1 con Síndrome de Van Der Woud y 1 con secuencia de Klippel-Feil (Tabla I). Así, la incidencia global de IVF fue del $14.4 \%$, pero si no tenemos en cuenta los pacientes sindrómicos que de base tienen mayor predisposición para esta alteración, tendríamos 13 pacientes con IVF que corresponden a una incidencia de IVF del 11\%.

El diagnóstico de IVF se realizó en promedio a los 5 años (3-7 años) de forma conjunta por Cirugía Plástica y Fonoaudiología mediante evaluación clínica, parámetros universales y videonasofaringoscopia. ${ }^{(9)}$ La evaluación del habla fue realizada por Fonoaudiología valorando la existencia de articulaciones compensatorias, emisión nasal y resonancia de la voz. Clínicamente se evaluó la longitud del paladar y su movilidad, así como la movilidad de las paredes faríngeas laterales y de la pared faríngea posterior. Para la confirmación de IVF, el mismo cirujano tratante llevó a cabo una nasofaringoscopia con nasoendoscopio flexible previa preparación del paciente y de su acompañante. ${ }^{(10)}$

La corrección de la IVF se realizó de forma interdisciplinaria, siguiendo la guía de atención integral de FISULAB de acuerdo a la anatomía y severidad del caso, del tal forma que lo primero que se realizó fue una verificación de la efectividad de la palatoplastia primaria y, de ser necesario, se realizó una veloplastia intravelar para lograr una adecuada reparación muscular. ${ }^{(11)}$

Así, de los 17 pacientes con IVF, 15 fueron llevados a una segunda cirugía a una edad promedio de 5.2 años (entre los 5 y los 7 años). A 13 se les realizó la técnica de palatoplastia descrita por Prada que consiste en un diseño de Z-plastia en la mucosa nasal y palatina en forma opuesta; y una veloplastia intravelar para reparación y reposicionamiento muscular. ${ }^{(1)} \mathrm{A}$ un paciente se le realizó de forma exclusiva una veloplastia intravelar y a otro faringoplastia de tipo Jackson. Dos pacientes aún tienen pendiente cirugía. Finalmente, de los 15 pacientes que fueron llevados a un segundo procedimiento quirúrgico, 10 resolvieron la IVF y lograron adecuados resultados en el habla confirmados por nasofaringoscopia, y 5 persistieron con alteraciones en el habla, por lo que se verificó la presencia de IVF en la nasofaringoscopia y se decidió realizar faringoplastia tipo Jackson en 3 de estos pacientes a los 9 años de edad promedio (Tabla II). Otro de los pacientes con IVF residual presentó hipernasali-

Tabla I. Características demográficas de la población de pacientes del estudio

\begin{tabular}{|c|c|c|c|c|c|c|c|}
\cline { 2 - 8 } \multicolumn{1}{c|}{} & \multirow{2}{*}{ Número de pacientes } & \multicolumn{2}{c|}{ Sexo } & \multicolumn{2}{c|}{ Clasificación } & \multicolumn{2}{c|}{ Etiología } \\
\cline { 2 - 8 } & & Masculino & Femenino & PH & LPH & Sindrómicos & No sindrómicos \\
\hline IVF & 17 & 8 & 9 & 2 & 15 & 4 & 13 \\
\hline No IVF & 101 & 59 & 42 & 13 & 88 & 15 & 86 \\
\hline Total & 118 & 67 & 51 & 15 & 103 & 19 & 99 \\
\hline
\end{tabular}

*PH: Paladar hendido, LPD: Labio paladar hendido, IVF: Insuficiencia velofaringea

Tabla II. Distribución de cantidad de pacientes con IVF según los procedimientos requeridos de corrección y cantidad de pacientes con IVF residual posterior a dichos procedimientos

\begin{tabular}{|c|c|c|c|c|c|c|c|c|}
\cline { 3 - 8 } \multicolumn{2}{c}{} & \multicolumn{5}{c|}{$\mathbf{1}^{\text {a Cirugía }}$} & $2^{\text {a Cirugía }}$ & \\
\hline $\begin{array}{c}\text { Pacientes } \\
\text { elegibles }\end{array}$ & $\begin{array}{c}\text { Pacientes } \\
\text { incluidos }\end{array}$ & $\begin{array}{c}\text { Pacientes } \\
\text { con IVF }\end{array}$ & Operados & $\begin{array}{c}\text { No } \\
\text { operados }\end{array}$ & $\begin{array}{c}\text { Con IVF } \\
\text { residual }\end{array}$ & Operados & $\begin{array}{c}\text { No } \\
\text { operados }\end{array}$ & $\begin{array}{c}\text { Con IVF } \\
\text { residual }\end{array}$ \\
\hline 972 & 118 & 17 & 15 & 2 & 5 & 3 & 2 & 0 \\
\hline
\end{tabular}

*IVF: Insuficiencia velofaringea 
dad leve con IVF del $10 \%$ en la nasoendoscopia, por lo que fue manejado únicamente con Terapia de Lenguaje y según su evolución clínica se decidirá la necesidad de nuevo procedimiento quirúrgico. Un paciente con IVF residual tiene pendiente cirugía próximamente.

Es importante resaltar que en los 5 pacientes que persistieron con alteraciones en el habla, el porcentaje de IVF reportado en la nasoendoscopia fue del $64 \%$ en promedio (20-90\%), que con la primera corrección quirúrgica de la IVF se redujo al 36\% (10-60\%) y al requerir un segundo procedimiento de corrección (faringoplastia), la IVF se redujo a un 5\% (0-5\%) según reporte de la nasoendoscopia (Tabla III).

\section{Discusión}

El diagnóstico temprano y el manejo interdisciplinario de la IVF son fundamentales para garantizar los buenos resultados del habla en pacientes con paladar hendido. Es importante la evaluación periódica de estos pacientes y su seguimiento temprano para detectar anomalías en el habla que permitan sospechar la presencia de IVF. ${ }^{(1,12)}$ La incidencia de IVF después de la primera palatoplastia se encuentra entre el 5-36\% en grupos institucionales de manejo interdisciplinario. En el presente estudio encontramos 118 pacientes tratados en FISULAB y seguidos durante los primeros 5 años de vida en dicha institución; de estos, el porcentaje global de IVF fue del $14.4 \%$. Sin embargo, de nuestros pacientes con IVF, un $23 \%$ corresponden a pacientes sindrómicos, lo que habla de la importante relación de la incidencia de IVF en pacientes sindrómicos con labio y paladar hendidos. Por lo tanto, al realizar la corrección del porcentaje de IVF sin los pacientes sindrómicos, encontramos que el porcentaje real de incidencia de IVF en nuestra población de pacientes fue del $11 \%$, dentro de los rangos de incidencia publicados en otras series. No hubo una diferencia significativa entre la presencia de IVF y el sexo de los pacientes.

La edad de la palatoplastia primaria fue principalmente entre los 8 y los 10 meses de edad, aunque en casos específicos se realizó de forma tardía dependiendo principalmente de la edad de ingreso del paciente en el programa. El porcentaje de éxito de la primera cirugía de paladar fue del $86 \%$, encontrándose dentro de los estándares mundiales del $80-90 \% .{ }^{(13,14)}$ la tasa de éxito con

Tabla III. Porcentaje de IVF reportado en la nasoendoscopia preoperatoria y postoperatoria de la primera y segunda corrección de IVF con los promedios parciales de los 5 pacientes que requirieron 2 procedimientos para corrección de la IVF y los promedios globales pre y postoperatorios de todos los pacientes con IVF

\begin{tabular}{|c|c|c|c|}
\hline Paciente & Preoperatoria & $\begin{array}{c}\text { Posterior a la } 1^{\mathrm{a}} \text { corrección } \\
\text { de IVF }\end{array}$ & $\begin{array}{c}\text { Posterior a la } 2^{\text {a }} \text { corrección } \\
\text { de IVF }\end{array}$ \\
\hline 1 & $50 \%$ & $40 \%$ & $0 \%$ \\
\hline 2 & $80 \%$ & $60 \%$ & $10 \%$ \\
\hline 3 & $90 \%$ & $40 \%$ & $5 \%$ \\
\hline 4 & $20 \%$ & $10 \%$ & Terapia \\
\hline 5 & $80 \%$ & $30 \%$ & Pendiente $\mathrm{cx}$ \\
\hline Promedio parcial & $64 \%$ & $36 \%$ & $5 \%$ \\
\hline 6 & $80 \%$ & $0 \%$ & \\
\hline 7 & $90 \%$ & $0 \%$ & \\
\hline 8 & $10 \%$ & $0 \%$ & \\
\hline 9 & $35 \%$ & $0 \%$ & \\
\hline 10 & $80 \%$ & $0 \%$ & \\
\hline 11 & $30 \%$ & $0 \%$ & \\
\hline 12 & $70 \%$ & $0 \%$ & \\
\hline 13 & $50 \%$ & $0 \%$ & \\
\hline 14 & $20 \%$ & $0 \%$ & \\
\hline 15 & $20 \%$ & $0 \%$ & \\
\hline 16 & $40 \%$ & Pendiente cx & \\
\hline 17 & $80 \%$ & Pendiente cx & \\
\hline $\begin{array}{c}\text { Promedio } \\
\text { total }\end{array}$ & $54.4 \%$ & $36 \%$ & $5 \%$ \\
\hline
\end{tabular}


Diagnóstico e intervención temprana de insuficiencia velofaringea posterior a palatoplastia primaria en pacientes atendidos interdisciplinariamente en FISULAB (Bogotá, Colombia)

la segunda cirugía fue del $66.6 \%$, y los 3 pacientes con IVF residual que fueron a una tercera cirugía de tipo faringoplastia resolvieron la IVF en un $100 \%$, sin tener en cuenta los 2 pacientes que no se operaron.

Con respecto a la corrección de la IVF, se emplearon diferentes técnicas quirúrgicas aunque en la mayoría de los casos se utilizó la técnica de palatoplastia de Prada, con veloplastia intravelar con Z-plastias opuestas en la mucosa nasal y palatina, logrando la mayor corrección en el porcentaje de IVF detectado en la nasoendoscopia y la resolución de la insuficiencia en la valoración clínica. Ante la necesidad de un segundo procedimiento por persistencia de IVF, lo cual ocurrió únicamente en 5 pacientes, la técnica quirúrgica más empleada fue la faringoplastia de Jackson, logrando con ella una resolución definitiva de la insuficiencia así como un porcentaje de IVF en la nasoendoscopia del 0 al 5\%, lo cual nos habla del gran impacto del primer procedimiento de corrección en la reducción del porcentaje de IVF en la nasoendoscopia y por lo tanto, de la importancia de verificar la efectividad de la palatoplastia primaria y corregirla, de ser necesario, antes de pasar a otros procedimientos como las técnicas de faringoplastia o los colgajos retrofaríngeos, ya que pueden ser obstructivos y asociarse al síndrome de apnea obstructiva del sueño. ${ }^{(15)}$

\section{Conclusiones}

La insuficiencia velofaríngea (IVF) requiere un diagnóstico y manejo interdisciplinarios, idealmente en edades previas al ingreso a la etapa escolar. La corrección quirúrgica de la IVF debe hacerse sobre una palatoplastia primaria adecuada, razón por la cual la primera cirugía de corrección de la IVF en la mayoría de los casos fue la técnica de palatoplastia de Prada. Cuando después de la reparación primaria persiste la IVF la corrección quirúrgica dependerá del patrón de cierre, siendo la faringoplastia de Jackson uno de los procedimientos más utilizados, en especial para casos leves a moderados. Con el presente estudio determinamos que la magnitud del defecto inicial se reduce principalmente después de la primera cirugía, ya que con la técnica utilizada se logra una adecuada reorientación y disposición muscular, produciendo así un mayor porcentaje de efectividad en el tratamiento de la IVF antes de pasar a otros procedimientos.

Verificamos una incidencia de IVF del $11 \%$ en nuestra población de pacientes, porcentaje que se encuentra dentro de los rangos publicados por otros grupos interdisciplinarios y que nos demuestra que lo más importante es procurar la efectividad en el procedimiento inicial de la palatoplastia primaria. Además nos permite ver la importancia del estricto seguimiento del paciente, en especial en sus primeros 5 años de vida, para realizar un diagnóstico y tratamiento oportuno de la IVF.

\section{Dirección del autor}

\author{
Dr. José Rolando Prada Madrid \\ FISULAB \\ Calle 161 a \#21-35 \\ Bogotá, Colombia \\ Correo electrónico: rpradam@gmail.com
}

\section{Bibliografía}

1. Prada R, Cardenas I, Echeverri MP. Detección y manejo temprano de la Insuficiencia velofaríngea: Una propuesta de manejo integral. Rev. Col.Cir.Plást. 2003; 9: 20-28.

2. Yamaguchi K, Lonic D.A Treatment Protocol for Velopharyngeal Insufficiency and the Outcome. Plast. Reconstr Surg. 2016; 138(2):290e-299e.

3. Echeverri MP, Prada R, Tavera MC. Insuficiencia Velofaríngea. En Coiffman F.: Cirugía Plástica, Reconstructiva y Estética. Ed. Amolca, Bogotá, Colombia, $3^{2}$ Ed. 2009; tomo 3, Pp.2300-2313.

4. Huang M.H. y col. Anatomic Basis of Cleft Palate and Velopharyngeal Surgery: Implications from a fresh cadaveric study. Plast. Reconstr Surg. 1998;101: 613-627.

5. Kriens O.B. Anatomy of the velopharyngeal area in cleft palate. Clin in Plast Surg.1975 2: 261-269.

6. Skolnick ML, McCall GN, Barnes M. The sphinteric mechanism of velopharyngeal closure. Cleft Palate J. 1973; 10:286305.

7. Croft CB, Shprintzen RJ, Ruben SJ. Patterns of Velopharyngeal valving in normal and Cleft Palate Subjects: a Multi-View Videofluoroscopic and Nasoendoscopic Study. Laryngoscope. 1981; 91: 265-271.

8. Prada Madrid JR., et al. Patrones de cierre velofaríngeo: Estudio comparativo entre población sana y pacientes con paladar hendido. Cir plást. Iberolatinoam, 2010; 36 (4): 305-312.

9. Henningsson G, Kuehn,D. Universal Parameters for Reporting Speech Outcomes in individuals with cleft palate. Cleft Palate-Cranio. J. 2008; 45(1):1-17.

10. Dalston R, Warren D, Dalston E. Use of Nasometry as a diagnostic tool for identifying patients with velopharyngeal impairment. Cleft Palate Craniofac J. 1991;28: 184-189.

11. Prada R, Echeverri MP. Insuficiencia Velofaríngea. En Prada R.: Cirugía Craneofacial. Edit. Impresión Médica, Bogotá, Colombia, 2012, Cap. 50, Pp.1103-1118.

12. Sadove M, Eppley B, Jones D. Velopharyngeal Insufficiency. En Bentz M, Pediatric Plastic Surgery. Edit. Appleton \&Lange, Pittsburg, Pen, 1998, Pp. 112-127.

13. Brothers DB, Dalston RW, Peterson HD and Lawrence WT. Comparison of the Furlow Double Opposing Z palatoplasty with the Wardill Kilner Procedure for isolated Clefts of the Soft Palate. Plast Reconstr Surg. 1995; 6 969-977.

14. Kirschner RE, Wang P, Jawad AF, et al. Cleft Palate Repair by Modified Furlow Double Opposing Z plasty: The Children's Hospital of Philadelphia Experience. Plast Recontr Surg. 1999; 104:1998-2014.

15. Blacam $\mathbf{C}$ et al. Surgery for Velopharyngeal Dysfunction: A Systematic Review of Interventions and Outcomes. Cleft Palate Craniofac J. 2018;3:405-422. 
